\title{
Performance evaluation of the geographic routing protocols scalability
}

\begin{abstract}
Scalability is an important design factor for evaluating the performance of routing protocols as the network size or traffic load increases. One of the most appropriate design methods is to use geographic routing approach to ensure scalability. This paper describes a scalability study comparing Secure Region Based Geographic Routing (SRBGR) and Dynamic Window Secure Implicit Geographic Forwarding (DWSIGF) protocols in various network density scenarios based on an end-to-end delay performance metric. The simulation studies were conducted in MATLAB 2106b where the network densities were varied according to the network topology size with increasing traffic rates. The results showed that DWSIGF has a lower end-to-end delay as compared to SRBGR for both sparse (15.4\%) and high density (63.3\%) network scenarios. Despite SRBGR having good security features, there is a need to improve the performance of its end-to-end delay to fulfil the application requirements.
\end{abstract}

Keyword: WSN; Geographic routing; End-to-end delay; SRGBR; DWSIGF 